J CIS/B-6 (1998 May, Chiba) 22p1-13

\title{
A Model of Phase Transition with Breaking Supersymmetry
}

\author{
H. Mashiyama and K. T. Mashiyama \\ Department of Physics, Faculty of Science, Yamaguchi University, \\ Yamaguchi 753-8512, Japan
}

(Received 22 March 1998)

A phenomenological model of free energy is presented to demonstrate a phase transition from a state with supersymmetry to a state without supersymmetry. The model is equivalent, in a commensurate phase, to the extended sublattice model for antiferroelectricity and ferrielectricity. In a selected set of parameters, the state with supersymmetry (antiferroelectric state) transforms to the symmetry broken state (ferrielectric state), with decreasing temperature. The transition is either first or second order one depending on the coefficients of the free energy.

Key wor ds: commensurate structure; additional symmetry operations; Landau theory of sublattice model

\section{INTRODUCTION}

It has been widely recognized that incommensurate phases are represented by $3+d$ dimensional space groups. ${ }^{[1]}$ The phase relations in the modulation wave are the additional degree of freedom. If the modulation period locks-into a multiple of the basic cell, the commensurate phase is realized, which should be one of the 230 space groups. The symmetry of a structure is reflected in the diffraction pattern, especially in the systematic absence of diffraction.

However, the diffraction pattern sometimes has a higher symmetry than that expected from the symmetry of the unit cell of the crystal. Such phenomena have been understood in terms of diffraction enhancement of symmetry. ${ }^{[2]}$ When the unit cell consists of subunits with which the scattered wave interferes, there is a definite relation between the subunits. Even if the relation is not a symmetry operation of space group, 
the unit cell is invariant under symmetry operations of so called groupoid. ${ }^{[3]}$

In our previous report, the additional symmetry, which relates subunits with each other within a superstructure (a commensurate structure induced from a high symmetry prototype phase), was called as supersymmetry. ${ }^{[4]}$ This symmetry operation was one of the element of the prototype phase. Generally the symmetry is lost at the protptype-to-superstructure transition (the incommensurate-to-commensurate transition). But it may exist in the commensurate phase, and exhibit higher symmetry of the diffraction pattern.

In this paper we propose a phenomenological model of free energy to describe supersymmetry. This model represents both incommensurate and commensurate phases. In the commensurate phase, the model is equivalent to the extended sublattice model for antiferroelectricity and ferrielectricity. ${ }^{[5]}$ By the use of the model, we conclude that a transition can take place between a structure with supersymmetry and a structure without supersymmetry. If we take no account of the supersymmetry and assign a usual space group, then the transition seems to accompany no symmetry change; a transition between structures with the same space group.

We describe briefly supersymmetry in the following section. Then the phenomenological free energy is introduced in sec. 3. The extreme of the free energy is calculated to discuss the phase transition. A discussion is given in sec. 4 .

\section{SUPERSYMMETRY IN A MODULATED STRUCTURE}

Let's consider a commensurate structure whose cell dimension is double along the c-axis of the prototype phase. The prototype space group is assumed to be Pmcn $(\mathrm{Z}=4)$ in order to discuss concretely. The modulated structure can be represented by modulation waves as shown in Fig. 1, where four chains of modulation exist corresponding to the number of formula unit.

In the incommensurate phase with $3+1$ dimensional space group Pmcn(ss-1), the following diffraction conditions exist;

$0 \mathrm{kl} \mathrm{m}$ : $l+m=$ even, $\quad h 0 l \mathrm{~m}: l+m=$ even and $h k 00: h+k=$ even.

Then the four chains in Fig. 1 are related with each other by 3+1 dimensional operations. In the commensurate phase, we refer Miller indexes $h_{\mathrm{s}} k_{\mathrm{s}} l_{\mathrm{s}}$ to the superlattice whose c axis is double the prototype cell. Then the above conditions are written as 


$$
0 k_{\mathrm{s}} l_{\mathrm{s}}: l_{\mathrm{s}}=\text { even, } \quad h_{\mathrm{s}} 0 l_{\mathrm{s}}: l_{\mathrm{s}}=4 \mathrm{n}, 4 \mathrm{n} \pm 1 \text { and } h_{\mathrm{s}} k_{\mathrm{s}} 0: h_{\mathrm{s}}+k_{\mathrm{s}}=\text { even. }
$$

Really these conditions are recognized in some $A_{2} B X_{4}$-type crystals. Although the first and third relations indicate the c- and n-glides, respectively, there is no space group that satisfies the second relation inevitably. The candidate of 3-dimensional space group for the superstructure is $\mathrm{P} 2{ }_{1} / \mathrm{c} 11$ or $\mathrm{Pc} 2{ }_{1} \mathrm{n}$. In Fig. 1 , the former space group relates chain 1 and 3 ( 2 and 4 ) by the proper symmetry operation. But there should not be any strict relation between 1 and 2 or 4 . On the other hand chain 1 and 4 ( 2 and 3) are related in the latter space group. And no relation exists between 1 and 2 or 3 .

It will be convinced that chain 1 and 2 are related with each other to satisfy the second reflection condition by an operation $\left\{\sigma_{y} \mid b / 2+c / 4\right\}$; an supersymmetry operation. Such symmetry is allowed within $3+\mathrm{d}$ dimensional space group if the modulation is incommensurate. However, the symmetry of the superstructure should belong one of 230 space groups. Then such a supersymmetry operation would be considered as an accidental relation. In order to demonstrate that supersymmetry can exist as a stable state, we examine a phenomenological model of free energy in the next section.

\section{SUBLATTICE MODEL OF SUPERSYMMETRY}

We start from the following free energy density describing the incommensurate-commensurate transition in $A_{2} B X_{4}$-type crystals ${ }^{[6]}$

$$
f=\alpha / 2|Q|^{2}+\beta / 4|Q|^{4}+\gamma / 6|Q|^{6}+\delta / 4\left(Q^{4}+Q^{* 4}\right)+i \sigma\left(Q \mathrm{~d}_{z} Q^{*}-Q^{*} \mathrm{~d}_{z} Q\right)+\kappa / 2\left|\mathrm{~d}_{z} Q\right|^{2},
$$

where $Q$ is a complex order parameter and $\mathrm{d}_{z}$ is a differential operator with respect to the spatial coordinate $z$. The last two terms in (3) are the Lifshitz invariant and the elastic energy term, respectively.

In the incommensurate phase, $|Q| \neq 0$ is a representative of the modulation amplitude. As noted in the previous section, all four waves from 1 to 4 in Fig. 1 have the same amplitude and each phase of the wave is related with others. In the commensurate phase, the phase of $Q$ locks-in a definite values. The coupling between $Q$ and polarization, strain or some other physical quantities works to lower the crystal system. Then two of four waves are related with each other. In other words, there are two subunits in the superstructure of Fig. 1; one unit is composed of chain 1 and 3, and other unit of chain 2 and 4, for example. These two units are independent in general.

In order to take the supersymmetry between subunits into account, a sublattice model 
is considered with an extension from the Kittel's model for antiferroelectricity. ${ }^{[7]}$ To describe two subunits, let's introduce two complex-order parameters $Q_{1}$ and $Q_{2}$, zone boundary modes in $A_{2} B X_{4}$-type incommensurate crystals. The free energy per unit volume is written as

$$
\begin{aligned}
F & =\alpha / 2\left(\left|Q_{1}\right|^{2}+\left|Q_{2}\right|^{2}\right)+\xi / 2\left(Q_{1} Q_{2}{ }^{*}+Q_{1}{ }^{*} Q_{2}\right)+\beta_{1} / 4\left(\left|Q_{1}\right|^{4}+\left|Q_{2}\right|^{4}\right)+\beta_{2} / 2\left|Q_{1}\right|^{2}\left|Q_{2}\right|^{2} \\
& +\beta_{3} / 4\left(Q_{1}{ }^{2} Q_{2}{ }^{* 2}+Q_{1}{ }^{* 2} Q_{2}{ }^{2}\right)+\beta_{4} / 2\left(Q_{1}{ }^{2} Q_{1}{ }^{*} Q_{2}{ }^{*}+Q_{1} Q_{2} Q_{2}{ }^{2 *}+Q_{1}{ }^{* 2} Q_{1} Q_{2}+Q_{1}{ }^{*} Q_{2}{ }^{*} Q_{2}{ }^{2}\right) \\
& +\delta_{1} / 8\left(Q_{1}{ }^{4}+Q_{2}{ }^{4}+Q_{1}{ }^{* 4}+Q_{2}{ }^{* 4}\right)+\delta_{2} / 4\left(Q_{1}{ }^{2} Q_{2}{ }^{2}+Q_{1}{ }^{* 2} Q_{2}{ }^{* 2}\right) \\
& +\delta_{3} / 2\left(Q_{1}{ }^{3} Q_{2}+Q_{1} Q_{2}{ }^{3}+Q_{1}{ }^{* 3} Q_{2}{ }^{*}+Q_{1}{ }^{*} Q_{2}{ }^{* 3}\right)+\gamma / 6\left(\left|Q_{1}\right|^{6}+\left|Q_{2}\right|^{6}\right) .
\end{aligned}
$$

The Lifshitz invariant and the elastic energy can be included in $\alpha$. Here both $Q_{1}$ and $Q_{2}$ belong to the same irreducible representation.

The free energy (4) has several extremes; a normal phase: $Q_{1}=Q_{2}=0$, a commensurate phase: $\left|Q_{1}\right|^{2}=\left|Q_{2}\right|^{2} \neq 0$, and another commensurate phase: $\left|Q_{1}\right|^{2} \neq\left|Q_{2}\right|^{2} \neq 0$. Though these two superstructures may belong to the same space group, the phase transition can take place with breaking supersymmetry.

Since we discuss about the commensurate structure, the free energy can also be written by two real order-parameters: $P_{1}$ and $P_{2}$. Then the free energy is identical with the extended sublattice model for antiferroelectricity introduced three decades ago; ${ }^{[8]}$

$$
\begin{aligned}
F= & \alpha / 2\left(P_{1}{ }^{2}+P_{2}{ }^{2}\right)+\xi P_{1} P_{2}+\beta / 4\left(P_{1}{ }^{4}+P_{2}{ }^{4}\right)+\eta\left(P_{1}{ }^{2}+P_{2}{ }^{2}\right) P_{1} P_{2} \\
& +\zeta / 2 P_{1}{ }^{2} P_{2}{ }^{2}+\gamma / 6\left(P_{1}{ }^{6}+P_{2}{ }^{6}\right) .
\end{aligned}
$$

Here the coefficients $\beta, \eta$ and $\zeta$ are redefined for the sake of simplicity.

Suzuki and Okada ${ }^{[5]}$ have discussed the case of $\beta<0, \xi>0$ and $\gamma>0$, and shown that the following sequence of transition takes place: paraelectric(non-polar $P_{1}=P_{2}=0$ ) phase $\rightarrow$ ferrielectric(semi-polar $P_{1} \neq P_{2} \neq 0$ ) phase $\rightarrow$ antiferroelectric(anti-polar $P_{1}=-P_{2} \neq 0$ ) phase. On the other hand, we select other set of parameters. Then the transition sequence is the following:

1) normal phase: $P_{1}=P_{2}=0$,

2) commensurate phase with supersymmetry(antiferroelectric): $P_{1}=-P_{2} \neq 0$,

3) commensurate phase without supersymmetry(ferrielectric): $P_{1} \neq-P_{2} \neq 0$. An example is shown in Fig. 2 for a set of selected parameters:

$$
\beta=2 \text { or }-4, \gamma=12, \xi=1, \eta=0, \zeta=16 .
$$

With decreasing temperature, normal phase 1 transforms at $\alpha=\xi$ into phase 2 with 
supersymmetry. The transition is a second order one, so far as the incommensurate phase is ignored. At lower temperature, phase 3 without supersymmetry is the minimum free energy state. The 2-to-3 transition is of second order $(\beta=2)$ or of first order $(\beta=-4)$. In the case of second-order transition, the free energy of phase 2 becomes a saddle point at

$$
\alpha=3 \xi / 2+(\beta+3 \zeta-10 \eta) / 8 \gamma\left[\left\{\beta-\zeta+2 \eta+\left[(\beta-\zeta+2 \eta)^{2}-8 \gamma \xi\right]^{1 / 2}\right\},\right.
$$

below which phase 3 appears. One of the principle values of the susceptibility matrix diverges at the transition. For other choice of parameters, other sequence of transition appears, e.g. $\mathrm{P}_{1}=-\mathrm{P}_{2} \neq 0 \rightarrow \mathrm{P}_{1} \neq \mathrm{P}_{2} \neq 0 \rightarrow \mathrm{P}_{1}=-\mathrm{P}_{2} \neq 0$ and $\mathrm{P}_{1} \neq \mathrm{P}_{2} \neq 0 \rightarrow \mathrm{P}_{1}=\mathrm{P}_{2} \neq 0$, even if $\xi>0$.

\section{DISCUSSION}

The phenomenological free energy (5) has explained that the state with supersymmetry is stable in a temperature range and that the transition takes place to the state without supersymmetry. So far a lock-in energy of $Q^{4}+Q^{* 4}$ has been considered in (3). But there may be a coupling between the primary order parameter $\mathrm{Q}$ and the macroscopic polarization $P$ or strain $S$; $\left(Q^{2}+Q^{* 2}\right) P$ or $\left(Q^{2}-Q^{* 2}\right) S$. These terms can stabilize a ferroelectric phase or a ferroelastic phase without supersymmetry.

In the commensurate phase 3 of $\mathrm{LiRbSO}_{4}$, the extra extinction rule has been recognized. ${ }^{[9]}$ If such an extinction rule holds exactly, then there should exist the symmetry operation in addition to the symmetry of space group. The phase 3 of $\mathrm{LiRbSO}_{4}$, in which the reflection conditions (2) hold, can be explained either monoclinic or orthorhombic space group as summarized below;

1) The space group is monoclinic $P 2_{1} / c$ and the supersymmetry $\left\{\sigma_{y} \mid b / 2 \pm c / 4\right\}$ exists.

2) The space group is orthorhombic $P c 2{ }_{1} \mathrm{n}$ and the supersymmetry $\left\{\sigma_{\mathrm{y}} \mid \mathrm{b} / 2 \pm \mathrm{c} / 4\right\}$ exists.

3) The extra extinction holds only approximately and the supersymmetry is not a strict relation. The crystal is ferroelectric $\mathrm{Pc} 2{ }_{1} \mathrm{n}$ or ferroelastic $\mathrm{P} 2{ }_{1} / \mathrm{c}$.

From the experimental point of view, the phase 3 of $\mathrm{LiRbSO}_{4}$ is considered to be ferroelastic $\mathrm{P} 2{ }_{1} / \mathrm{c}^{[10]}$ However, the model free energy (4) indicates that the supersymmetry can be allowed in the phenomenological theory. And there is a possibility that the phase with supersymmetry transforms into the usual space group 
phase with breaking the supersymmetry. In other commensurate phase, there is a possibility that supersymmetry exists potentially. ${ }^{[4,11]}$

\section{REFERENCES}

[1] P. M. de Wolff, T. Janssen and A. Janner, Acta Cryst. A 37, 625 (1981).

[2] H. Iwasaki, Acta Cryst. A 28, 253 (1972).

[3] A. Yamamoto and K. N. Ishihara, Acta Cryst. A 44, 707 (1988).

[4] H. Mashiyama, Proc. of IMF-9 (1997, Seoul), J. Korean Phys. Soc. 32, S877 (1998).

[5] I. Suzuki and K. Okada, J. Phys. Soc. Jpn. 45, 1302 (1978).

[6] See for example, Y. Ishibashi, Incommensurate Phases in Dielectrics 2 , ed. by R. Blinc and A. P. Levanyuk (North-Holland, Amsterdam,1986) Chap.11.

[7] C. Kittel, Phys. Rev. 82, 729 (1951) .

[8] L. Benguigui, Can. J. Phys. 46, 1627 (1968).

[9] H. Mashiyama, K. Hasebe, S. Tanisaki, Y. Shiroishi and S. Sawada, J. Phys. Soc. Jpn. 47, 1198 (1979).

[10] A. Kunishige and H. Mashiyama, J. Phys. Soc. Jpn. 56, 3189 (1987).

[11] H. Shigematsu, T. Matsui, H. Mashiyama and Y. Noda, Proc. of IMF-9 (1997, Seoul), J. Korean Phys. Soc. 32, S169 (1998). 


\section{Figure captions}

FIGURE. 1 The modulation waves in the commensurate phase. The cell dimension along $\mathrm{z}$ is double the normal phase. Dark and light gray circles represent the same kind of atoms. The y- and z- coordinates are also modulated, though they are not shown explicitly.

FIGURE. 2 Temperature $(\alpha)$ dependence of order parameters for the extended sublattice model. Two order parameters $P_{1}$ and $P_{2}$ appears if $\alpha<\xi=1$. For $\beta=-4$, the transition at $\alpha=0.3404$ is of first order, which is indicated by arrows. If $\beta=2$, the second-order transition takes place at $\alpha=-7 / 12$, where other coefficients are $\gamma=12, \eta=0$ and $\zeta=16$. 
FIGURE. 1 The modulation waves in the commensurate phase. The cell dimension along $\mathrm{z}$ is double the normal phase. Dark and light gray circles represent the same kind of atoms. The y- and z- coordinates are also modulated, though they are not shown explicitly.

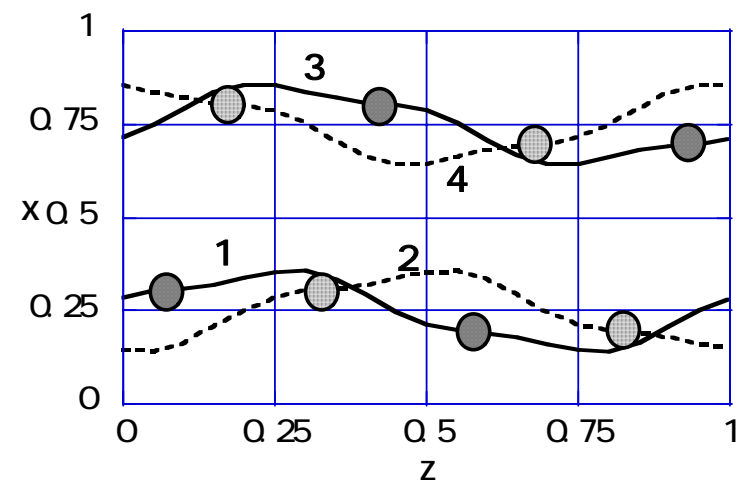

FIGURE. 2 Temperature $(\alpha)$ dependence of order parameters for the extended sublattice model. Two order parameters $P_{1}$ and $P_{2}$ appears if $\alpha<\xi=1$. For $\beta=-4$, the transition at $\alpha=0.3404$ is of first order, which is indicated by arrows. If $\beta=2$, the second-order transition takes place at $\alpha=-7 / 12$, where other coefficients are $\gamma=12, \eta=0$ and $\zeta=16$.

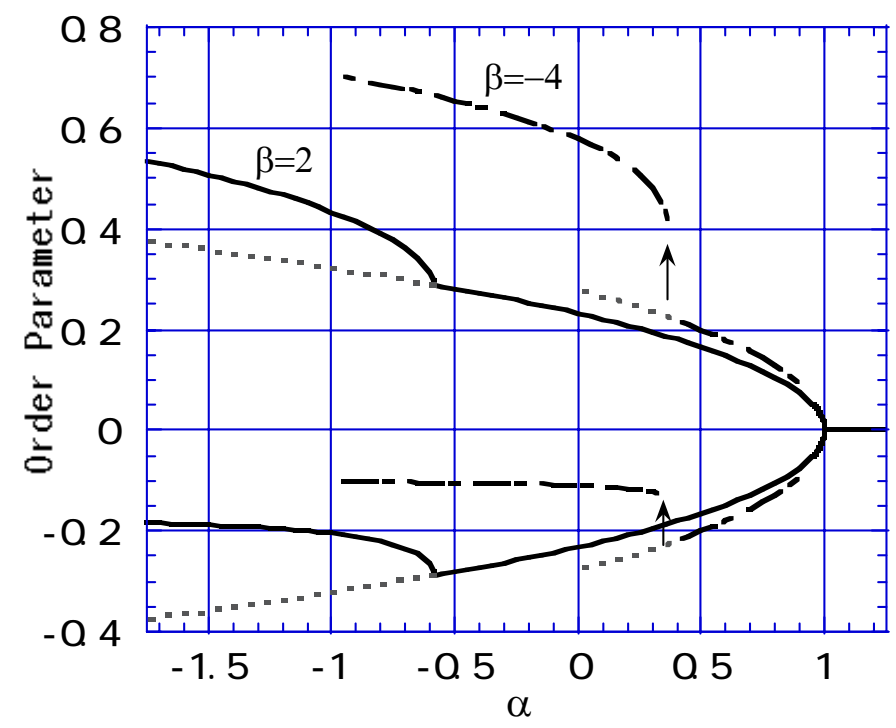

